\title{
CD147 promotes the proliferation, invasiveness, migration and angiogenesis of human lung carcinoma cells
}

\author{
SHAOXING YANG ${ }^{1 *}$, FEI QI $^{2 *}$, CHUANHAO TANG $^{1}$, HONG WANG $^{1}$, HAIFENG QIN $^{1}$, \\ XIAOYAN LI ${ }^{1}$, JIANJIE LI ${ }^{1}$, WEIXIA WANG ${ }^{1}$, CHANGYUN ZHAO $^{1}$ and HONGJUN GAO ${ }^{1}$ \\ ${ }^{1}$ Department of Pulmonary Oncology, Affiliated Hospital, Academy of Military Medical Science, Beijing 100071; \\ ${ }^{2}$ Department of Respiratory Medicine, Chinese PLA General Hospital, Beijing 100853, P.R. China
}

Received March 16, 2015; Accepted May 5, 2016

DOI: $10.3892 / 01.2016 .5502$

\begin{abstract}
Cluster of differentiation (CD) 147 is a transmembrane glycoprotein that is highly expressed at the tumor cell surface, which stimulates fibroblasts to produce a large number of matrix metalloproteinases and promotes tumor invasion and metastasis and tumor-induced angiogenesis. The present study investigated the functions and the role of CD147 in the human lung carcinoma A549 cell line. The present study constructed expression and interference [small interfering (si) RNA] lentiviral vectors of CD147, which established stable overexpression and low expression of CD147 in the A549 cell line, named A549-CD147 and A549-siCD147, respectively. The differences in biological features between various levels of CD147 expression in A549 cells was investigated by cell counting kit-8 (CCK-8), Transwell, scratch and lumen formation assays. The results of the CCK- 8 assay revealed that A549-CD147 cell proliferation was significantly increased and A549-siCD147 cell proliferation was decreased compared with the control groups. The A549-CD147 cells had the largest number of cells penetrating the Matrigel in the Transwell assay, which indicates that upregulation of CD147 expression increases the infiltration capacity of cells. The scratch assay revealed that A549-CD147 cells have the highest capacity for migration, while A549-siCD147 cells have the lowest. Quantitative polymerase chain reaction and western blot analysis demonstrated that vascular endothelial growth factor (VEGF) expression was proportional to the expression level of CD147 at the mRNA and protein level. The lumen formation assay revealed that the number of vessel lumens that human umbilical vein endothelial cells formed in the A549-CD147 cell supernatant was increased compared
\end{abstract}

Correspondence to: Mr. Hongjun Gao, Department of Pulmonary Oncology, Affiliated Hospital, Academy of Military Medical Science, 8 East Avenue, Fengtai, Beijing 100071, P.R. China

E-mail: gaohj6708@sina.com

*Contributed equally

Key words: CD147, A549, malignant, tumor, lentiviral with the A549-siCD147 cells. Collectively, the present results suggest that CD147 is important in the promotion of lung carcinoma cell proliferation, invasion and metastasis and the upregulation of VEGF, which stimulates the angiogenesis of lung carcinoma. In conclusion, CD147 may be a potential target in the treatment of lung carcinoma.

\section{Introduction}

Malignant tumors are a fatal disease that threaten human health, due to their rapid growth and biologically progressive characteristics, lack of effective means for early diagnosis, high mortality rates and poor prognosis. Lung cancer causes the most morbidity and mortality out of all types of malignant tumors (1), of which non-small cell lung cancer accounts for $80 \%$. Adenocarcinoma, squamous cell carcinoma and large cell lung cancer cause morbidity and mortality in a descending order. Lung adenocarcinoma may transform into hematogenous metastasis, and an increasing proportion of patients with this type of cancer have been observed in recent studies (2). In addition, lung adenocarcinoma has become the most common disease for women and younger patients; therefore, in depth research of its biological characteristics is essential (2).

Cluster of differentiation (CD) 147, also known as extracellular matrix metalloproteinase inducer, was originally derived from human lung cancer LX-1 cell line, and belongs to the immunoglobulin super-family (3). It stimulates fibroblasts to produce matrix metalloproteinases (MMPs) (4-8), which control basement membrane components and the remodeling of extracellular matrix macromolecules, promote tumor invasion and metastasis and induce tumor angiogenesis (9-11). Keratinocytes primarily express low levels of CD147 in normal cells of the thymic epithelium as do retinal pigment epithelial cells, but in malignant tumors CD147 expression is often increased, and has been observed in lung, ovarian and liver cancer cells (12-14).

Numerous studies have demonstrated that CD147 is a key molecule in various physiological or pathological processes. Although its function has been investigated in various studies, the role of CD147 has not been previously demonstrated in lung cancer cells, to the best of our knowledge. In the present study, the function and role of CD147 in human lung carcinoma A549 cells is investigated. 


\section{Materials and methods}

Cell lines. Human lung carcinoma A549 cells, 293FT cells human infant foreskin fibroblasts (HFF) cells and human umbilical vein endothelial cells (HUVEC) were obtained from China Infrastructure of Cell Line Resources (Chinese Academy of Medical Sciences, Beijing, China), cultured in $\mathrm{H}$-DMEM added with $10 \% \mathrm{FBS}$ and maintained at $37^{\circ} \mathrm{C}$ in a $5 \% \mathrm{CO}_{2}$ incubator. DH5 $\alpha^{\mathrm{TM}}$ Competent Cells was purchased from Thermo Fisher Scientific, Inc. (Waltham, MA, USA).

Construction of CD147 expression vector. CD147 primers were designed according to the full-length sequences and restriction sites identified by GenBank (www.ncbi.nlm.nih.gov/genbank/) and synthesized by Invitrogen ${ }^{\mathrm{TM}}$ (Thermo Fisher Scientific, Inc.), as follows: CD147, sense 5'-GCTAGCATCATGGCG GCTGCGCTGTT-3' and antisense 5'-TCTAGAGGAAGA GTTCCTCTGGCGGACGTT3-'. Total mRNA was extracted from A549 cells using Trizol (Invitrogen ${ }^{\mathrm{TM}}$; Thermo Fisher Scientific, Inc.), and subsequently $1 \mu \mathrm{g}$ was used to obtain the full-length sequence of CD147 using the primers mentioned above using reverse transcription-polymerase chain reaction (RT-PCR). Using PrimeScriptTM RT reagent Kit (TaKaRa, Japan) and PrimeScriptTM PCR reagent Kit (TaKaRa, Japan). PCR conditions were pre-denaturation at $94^{\circ} \mathrm{C}$ for $5 \mathrm{~min}$, denaturation at $94^{\circ} \mathrm{C}$ for $30 \mathrm{sec}$, primer annealing at $57^{\circ} \mathrm{C}$ for $30 \mathrm{sec}$, and extension at $72^{\circ} \mathrm{C}$ for $90 \mathrm{sec}$, for 30 cycles, with a final extension step at $72^{\circ} \mathrm{C}$ for $4 \mathrm{~min}$. The whole CD147 gene was recombined into a PMD-vector (ABigen Corporation, Beijing, China), and transformed into Escherichia coli (ABigen Corporation). Following blue-white screening, positive (white) clones were selected and multiplied in LB culture medium (Caisson Laboratories, Logan, UT, USA). The recombinant plasmid, which was verified by sequencing (Invitrogen ${ }^{\mathrm{TM}}$; Thermo Fisher Scientific, Inc.), and pEGFP vectors (ABigen Corporation) were double digested by XbaI and Nhe II (Takara Bio, Inc., Otsu, Japan). The target gene and vector fragment were recovered and ligated using the Quick Connect kit (Takara Bio, Inc.) at $16^{\circ} \mathrm{C}$ overnight. Subsequently, the plasmid was transformed into E. coli . The positive clones were multiplied in LB culture medium, and the plasmids were extracted for PCR, enzyme digestion and sequencing identification. The verified CD147 overexpression vector was named pegfp-CD147.

Construction of CD147 RNA interference vector. The interference vectors were designed (using GenBank) against the $19 \mathrm{bp}$ RNA sequence fragment and the corresponding off-target control sequence, which was in compliance with the human CD147 gene for RNA interference characteristics and the pSicoR-GFP lentiviral insertion sequence requirements. Small interference (si) vector sequences were synthesized by (Invitrogen $^{\mathrm{TM}}$; Thermo Fisher Scientific, Inc.) as follows: siRNA vector 1, sense 5'-GATCCGTGACCAAGAACGTCT TCAAGAGAGACGTTCTTGCCTTTGTCATTTTCCTTT TTTC-3' and antisense 5'-AGCTTTTCCAAAAAATGACAA AGGCAAGAACGTCTCTCTTGAAGACGCCTTTTTTC-3'; siRNA vector 2 , sense 5'-GATCCACTACCGTTGTTATAG GTGTTCAAGAGACACCTATAACAACGGTAGTTTTTT TGGAAA-3' and antisense 5'-AGCTTTTCCAAAAAAACT ACCGTTGTTATAGGTGTCTCTTGAACACCTATAACA
ACGGTAGTG-3'; siRNA vector 3, sense 5'-TGGCTGTGA AGTCGTCAGAATTCAAGAGATTCTGACGACTTCACA GCCTTTTTTC-3' and antisense 5'-TGGCTGTGAAGTCGT CAGAATTCAAGAGATTCTGACGACTTCACAGCCTTT TTTC-3'. The pSicoR-GFP vector (ABigen Corporation) was double digested with the restriction enzymes XhoI and HpaI (Takara Bio, Inc.). Following 2\% gel electrophoresis, the vector DNA was recovered and transformed with the interference lentiviral vectors with the interference sequence synthesized through annealing, phosphorylation and ligation reaction. Subsequently, the interference lentiviral vectors were transformed using the heat stroke method into DH5 $\alpha$ competent cells and verified by sequencing.

Lentivirus packaging. Lentivirus packing 293FT cells were cultured in high glucose-Dulbecco's modified Eagle medium (H-DMEM; Gibco ${ }^{\circledR}$; Thermo Fisher Scientific, Inc.) with $10 \%$ fetal bovine serum (FBS; Hyclone ${ }^{\mathrm{TM}}$; GE Healthcare, Chalfont, UK). In total, $5 \mu \mathrm{g}$ target plasmids (pEGFP-CD147, pEGFP, pSicoR-siCD147, pSicoR-GFP), $4.2 \mu \mathrm{g}$ pLP, $2 \mu \mathrm{g}$ $\mathrm{pLP} 2$ and $2.8 \mu \mathrm{g} \mathrm{pLP} / \mathrm{VSVG}$ plasmids were used to transfect 293FT cells $\left(10^{6}\right.$ cells $)$. After $6 \mathrm{~h}$, novel media replaced the medium, which contained $1 \mathrm{mmol} / 1$ sodium pyruvate. The culture supernatant was collected after $48 \mathrm{~h}$, filtered with a $0.45 \mathrm{uM}$ filter (EMD Millipore, Billerica, MA, USA), ultracentrifuged, aliquoted and stored at $-80^{\circ} \mathrm{C}$.

Infection of A549 cells with lentivirus. A549 cells were cultured in H-DMEM with 10\% FBS. When the cells reached a $60 \%$ density, the medium was removed and $1 \mathrm{ml}$ of four lentiviral plasmids were added (pEGFP-CD147, pEGFP, pSicoR-siCD147, pSicoR-GFP) with Lipofectamine 2000 (Invitrogen $^{\mathrm{TM}}$; Thermo Fisher Scientific, Inc.) and $8 \mathrm{mg} / \mathrm{l}$ Polybrene (Sigma-Aldrich, St. Louis, MO, USA), and the cells were incubated at $37^{\circ} \mathrm{C}$ in a $5 \% \mathrm{CO}_{2}$ incubator overnight. The next day, the lentiviruses were removed by aspiration of the medium and complete medium was added. When the cells reached $80-90 \%$ density, a passage of 1:3 was performed. Subsequently, the cells were sorted according to the expression of green fluorescent protein (GFP) by flow cytometry (Guava ${ }^{\circledR}$; EMD Millipore), and cells were designated as A549-siCD147 or A549-GFP.

Extraction of total mRNA and RT-quantitative PCR (qPCR). Total cellular mRNA was extracted by Trizol, according to the manufacturer's instructions. Subsequently, $1 \mu \mathrm{g}$ mRNA was reverse transcribed into cDNA using PrimeScript ${ }^{\mathrm{TM}} \mathrm{RT}$ Reagent kit with gDNA Eraser (Takara Bio, Inc.), according to the manufacturer's protocol. The expression of CD147 and VEGF was detected using qPCR with the $\mathrm{iQ}^{\mathrm{TM}} 5$ Real-Time PCR Detection System (Bio-Rad Laboratories, Inc., Hercules, CA, USA). Glyceraldehyde 3-phosphate dehydrogenase (GAPDH) was used as an internal reference. The primers were designed by Primer Premier 5.0 (www.premierbiosoft. com/primerdesign/) and synthesized by Invitrogen ${ }^{\mathrm{TM}}$ (Thermo Fisher Scientific, Inc.), as follows: CD147, sense 5'-GCTAGC ATCATGGCGGCTGCGCTGTT-3' and antisense 5'-TCT AGAGGAAGAGTTCCTCTGGCGGACGTT-3'; VEGF, sense 5'-ATGCGGATCAAACCTCACCA-3' and antisense, 5'-TTACACGTCTGCGGATCTTG-3'; GAPDH, sense 5'-GAG TCAACGGATTTGGTCGT-3' and antisense 5'-TTGATTTTG 
GAGGGATCTCG-3'. PCR conditions were as follows: Pre-denaturation at $94^{\circ} \mathrm{C}$ for $5 \mathrm{~min}$, denaturation at $94^{\circ} \mathrm{C}$ for $30 \mathrm{sec}$, annealing at $57^{\circ} \mathrm{C}$ for $30 \mathrm{sec}$, and extension at $72^{\circ} \mathrm{C}$ for $90 \mathrm{sec}$ for 30 cycles, with a final extension step at $72^{\circ} \mathrm{C}$ for $4 \mathrm{~min}$. The $\mathrm{Cq}$ method was used for normalization (15).

Western blot analysis. Cell protein was extracted from the cells using radioimmunoprecipitation buffer (Shanghai Amquar Biological Technology Co., Ltd, Shanghai, China). In total, $200 \mu \mathrm{g}$ protein was used for sodium dodecyl sulfate polyacrylamide gel electrophoresis (15\% agarose). The gel was transferred to a polyvinylidene difluoride membrane, which was blocked for $1 \mathrm{~h}$ with $5 \%$ skim milk at room temperature and incubated with mouse monoclonal anti-CD147 (catalog no., sc-374101; dilution, 1:300) or rabbit polyclonal $\beta$-actin (catalog no., sc-130656; dilution, 1:1,000) antibodies (Santa Cruz Biotechnology, Inc., Dallas, TX, USA) at $4^{\circ} \mathrm{C}$ overnight. The membrane was washed with Tris-buffered saline with Tween 20 (TBST) three times for $10 \mathrm{~min}$ each, and incubated with hydrogen peroxidase (HRP)-conjugated goat anti-mouse and goat anti-rabbit secondary antibodies (catalog nos., sc-2301 and sc-2030, respectively; dilution, 1:1,000 and 1:2,000, respectively; Santa Cruz Biotechnology, Inc.) at room temperature for $1 \mathrm{~h}$, washed with TBST three times for $15 \mathrm{~min}$ each, and finally developed using enhanced chemiluminescence (Pierce ${ }^{\mathrm{TM}}$ ECL Plus Western Blotting Substrate; Thermo Fisher Scientific, Inc.). $\beta$-actin was used as internal reference.

Cell proliferation assay. Cell proliferation was assayed using a cell counting kit-8 (CCK-8) assay. The four types of cells (A549-CD147, A549-pEGFP, A549-siCD147, A549-pSicoR) were seeded in 96-well plates ( $\sim 1 \times 10^{4}$ cells/well or $200 \mu$ l cells) and cultured. Time points of $0,24,48,72 \mathrm{~h}$ were selected. The medium was removed after culturing and $100 \mu \mathrm{l}$ medium and $10 \mu$ l CCK-8 (Dojindo Molecular Technologies, Inc., Kumamoto, Japan) was added to each well. The cells were further cultured for $2 \mathrm{~h}$ at $37^{\circ} \mathrm{C}$ and $5 \% \mathrm{CO}_{2}$. Subsequently, a microplate reader was used to detect the optical density values at a $450 \mathrm{~nm}$ wavelength.

Immunofluorescence. Cells were seeded in 48-well plates at room temperature with $4 \%$ paraformaldehyde for $20 \mathrm{~min}$, then washed 3 times for $5 \mathrm{~min}$ each time in phosphate-buffered saline (PBS). PBS containing $10 \%$ rabbit serum $\left(\mathrm{Gibco}^{\circledR}\right.$; Thermo Fisher Scientific, Inc.) was used for blocking for $30 \mathrm{~min}$, followed by incubation with mouse monoclonal anti-CD147 (catalog no., sc-46700; dilution, 1:100; Santa Cruz Biotechnology, Inc.) and goat polyclonal anti-vascular endothelial growth factor (VEGF; catalog no., AF-293-SP; dilution, 1:200; R\&D Systems, Inc., Minneapolis, MN, USA) primary antibodies at $4^{\circ} \mathrm{C}$ overnight. The cells were washed with PBS three times for 5 min each, followed by incubation with fluorescein isothiocyanate- or tetramethylrhodamine-labeled anti-rabbit secondary antibodies (cat no. F0112, R\&D Systems, Inc., dilution, 1:100) at room temperature for $30 \mathrm{~min}$. Subsequently, the cells were washed with PBS three times for 5 min each, stained with 4',6-diamidino-2-phenylindole (DAPI; Dojindo Molecular Technologies, Inc.) and observed and photographed using an inverted fluorescence microscope (Olympus IX71; Olympus Corporation, Tokyo, Japan).
Scratch assay. Cells were seeded in 6-well plates $\left(5 \times 10^{5}\right.$ cells/well) overnight. A scratch was made in the wells using a pipette tip. The cells were washed three times with PBS and were photographed (TS100; Nikon Corporation, Tokyo, Japan) at 24, 48, 7h and $96 \mathrm{~h}$.

Migration assay. Matrigel (BD Biosciences, Franklin Lakes, NJ, USA) was melted at $4^{\circ} \mathrm{C}$ overnight and was diluted with serum-free medium which was pre-cooled at $4^{\circ} \mathrm{C}$ to a final concentration of $1 \mathrm{mg} / \mathrm{ml}$. In total, $100 \mu \mathrm{l}$ diluted Matrigel was added to the bottom chamber of a Transwell plate (EMD Millipore), and incubated at $37^{\circ} \mathrm{C}$ for $4-5 \mathrm{~h}$ to dry. Subsequently, 600-800 $\mu 1$ RPMI-1640 (Gibco ${ }^{\circledR}$; Thermo Fisher Scientific, Inc.) containing $10 \%$ serum was added to the bottom chamber and $2 \times 10^{4}$ cells were added to the Transwell plate. The plate was cultured in an incubator for $24 \mathrm{~h}$. Subsequently, the supernatant was removed and $800 \mu 1$ methanol was added to fix the cells for $30 \mathrm{~min}$ at room temperature. The fixed cells were blotted dry and stained with Giemsa (Beijing Solarbio Science \& Technology Co., Ltd., Beijing, China) for 15-30 min at room temperature. The stained cells were counted in nine random fields under the microscope (TS100; Nikon Corporation).

Lumen formation assay. In total, $60 \mu 1$ Matrigel was added to each well of a 96-well plate, and the plate was agitated. When the Matrigel solidified, $100 \mu$ l HUVEC, which were at the logarithmic phase, and four types of cell supernatant (A549-CD147, A549-pEGFP, A549-siCD147, A549-pSicoR) were added to the plate and cultured at $37^{\circ} \mathrm{C}$ and $5 \% \mathrm{CO}_{2}$ for $16 \mathrm{~h}$. The formation of lumens in each group of cells was observed using a phase contrast microscope (TS100; Nikon Corporation).

Statistical analysis. SPSS version 22.0 (SPSS Inc., Chicago, IL, USA) was used for analysis. Student's T test was used to analyze differences and $\mathrm{P}<0.05$ was considered to indicate a statistically significant difference.

\section{Results}

Enhanced expression of CD147 and VEGF in human lung carcinoma A549 cells. As shown in Fig. 1A and B, qPCR demonstrated that CD147 expression was enhanced in A549 cells compared with HUVEC and HFF cells. The expression of VEGF was also upregulated in A549 cells compared with HUVEC and HFF cells.

Co-localization of VEGF with CD147 in A549 cells. To investigate whether there was an association between CD147 and VEGF in A549 cells, the cells were co-labeled with anti-CD147 and anti-VEGF antibodies, stained with DAPI and observed under an immunofluorescence microscope. In the plasma membrane, VEGF co-localized with CD147 in A549 cells (Fig. 1C).

siRNA-mediated silencing and upregulation of CD147 expression. qPCR and western blot analysis were performed to investigate the expression of CD147 in A549-pSicoR, A549-siCD147, A549-pEGFP and A549-CD147 cells (Fig. 2B and C). Transfection with of A549 cells with CD147-siRNA resulted in a marked decrease of CD147 expression $(\mathrm{P}=0.0087)$. Transfection with A549-CD147 resulted in a 
A

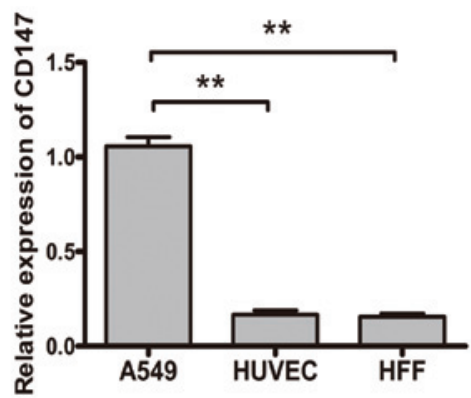

C
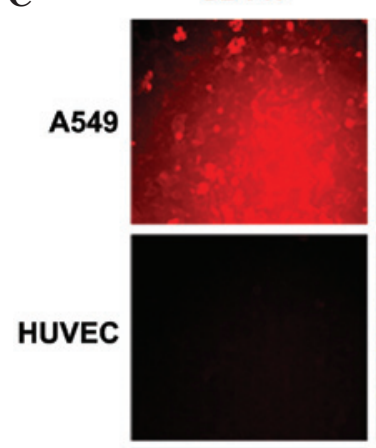

HFF

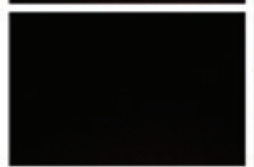

B

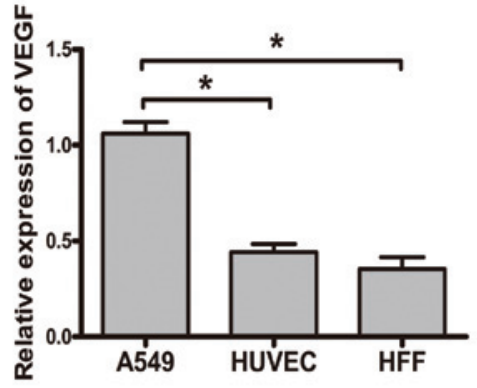

DAPI
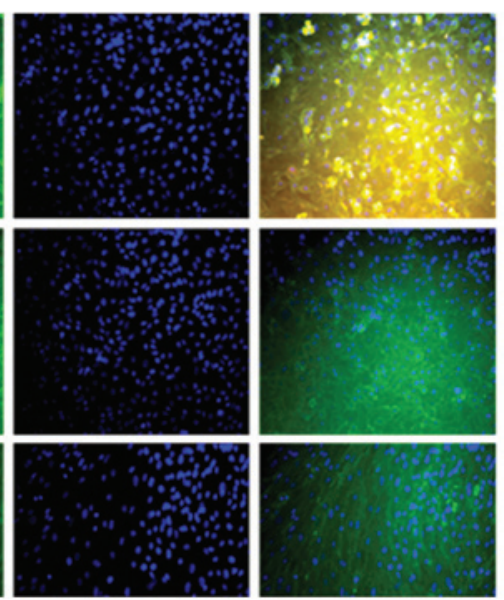

Figure 1. Expression of (A) CD147 and (B) VEGF in human lung carcinoma A549 cells, HUVEC and HFF, as detected using quantitative polymerase chain reaction. (C) CD147 co-localized with VEGF in the plasma membrane of A549 cells as detected by immunofluorescence. "P<0.05; ** $<<0.01$. CD, cluster of differentiation; VEGF, vascular endothelial growth factor; HUVEC, human umbilical vein endothelial cells; HIFF, human infant foreskin fibroblasts; DAPI, 4',6-diamidino-2-phenylindole.

A

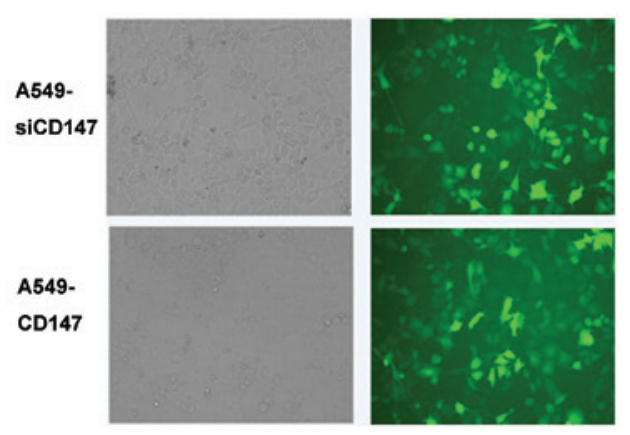

B

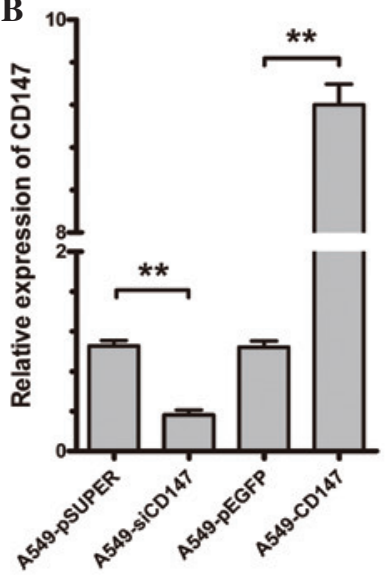

C

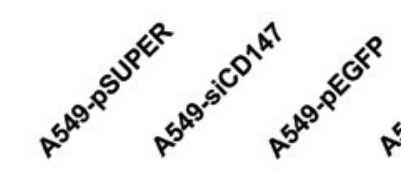

CD147

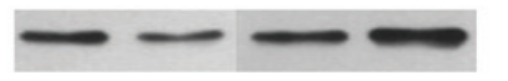

$\beta$-actin

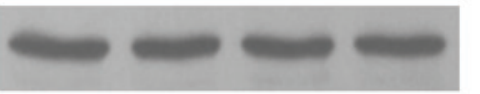

Figure 2. (A) Cell culture of human lung carcinoma A549-siCD147 and A549-CD147 cells. (B and C) Expression of CD147 in A549-CD147, A549-pEGFP, A549-siCD147 and A549-pSicoR cells as detected by (B) quantitative polymerase chain reaction and (C) western blot analysis. ${ }^{* *} \mathrm{P}<0.001$. CD, cluster of differentiation; si, small interfering.

clear increase of CD147 expression in A549 cells ( $\mathrm{P}=0.0064)$. By contrast, transfection with empty vectors (pSicoR and p-EGFP) did not interfere with CD147 expression at either the mRNA or protein level in A549 cells.

Effects of CD147 on the proliferation, invasiveness and migration of A549 cells. The effects of CD147 silencing on cell proliferation, invasiveness and migration was investigated in various cell lines. As shown in Fig. 3A, the proliferation of A549-CD147 cells was significantly increased compared with A549-pEGFP cells $(\mathrm{P}=0.0093)$. Compared with the proliferation of A549-pSicoR cells, the proliferation of A549-siCD147 was significantly inhibited to $37.73 \%$ $(\mathrm{P}=0.0325), 48.04 \%(\mathrm{P}<0.001)$, and $44.72 \%$ at 24,48 and $72 \mathrm{~h}$, respectively. A Transwell invasion assay using Matrigel-coated filters revealed that invasiveness was significantly increased in 
A

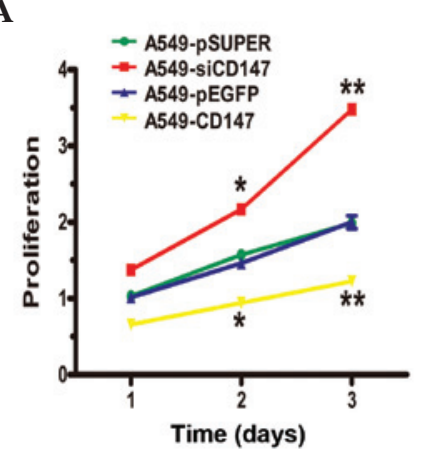

B

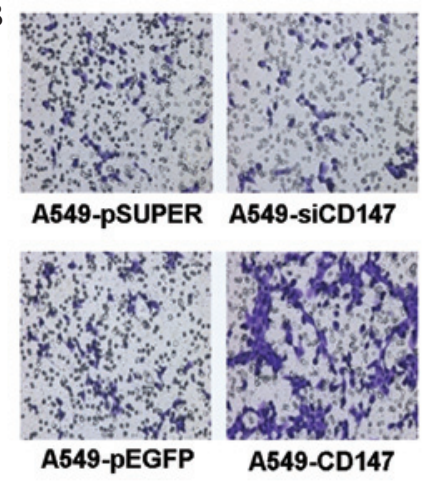

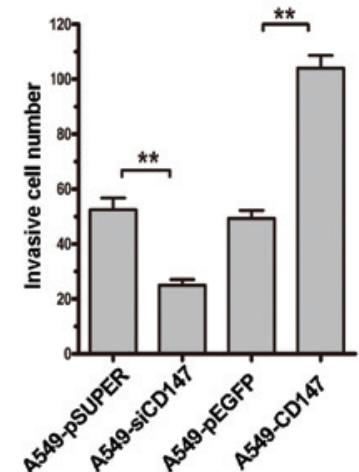

C
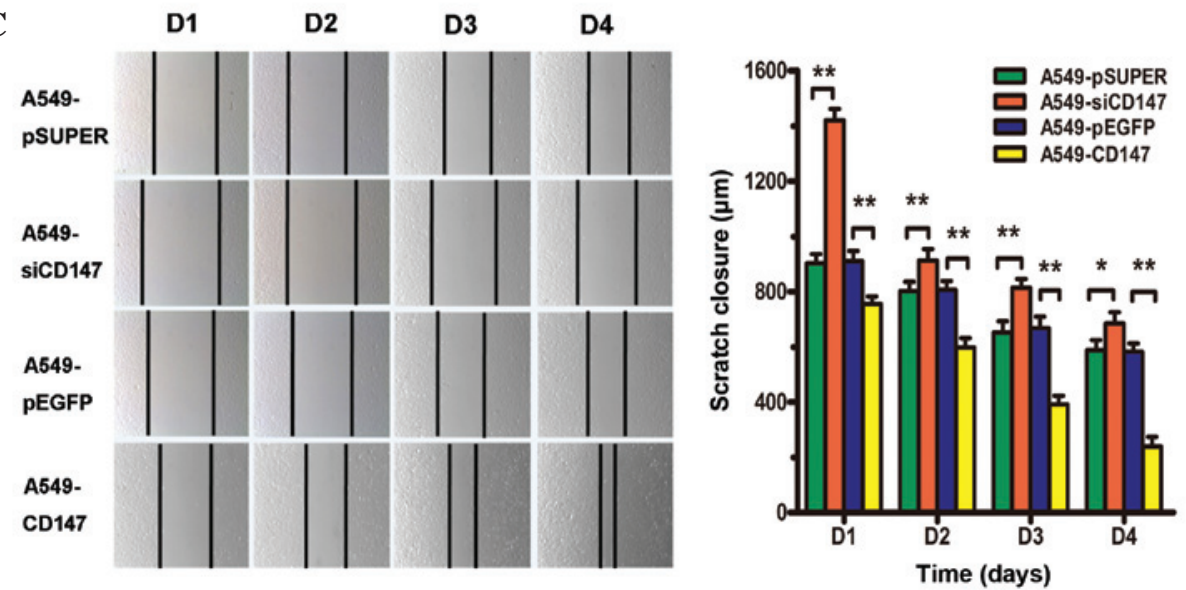

Figure 3. (A) Proliferation of human lung carcinoma A549-CD147, A549-pEGFP, A549-siCD147 and A549-pSicoR cells, as detected by cell counting kit-8 assay. (B) Invasiveness of A549-CD147, A549-pEGFP, A549-siCD147 and A549-pSicoR cells as detected by Transwell assay. (C) Migration of A549-CD147, A549-pEGFP, A549-siCD147 and A549-pSicoR cells as detected by scratch assay. ${ }^{*} \mathrm{P}<0.05 ;{ }^{* *} \mathrm{P}<0.01$. CD, cluster of differentiation; D, day.

A549-CD147 cells compared with A549-pEGFP A549 cells, and was inhibited in A549-siCD147 cells compared with A549-pSicoR cells (Fig. 3B). The scratch test results revealed that the migration of A549-CD147 cells was significantly increased and the migration of A549-siCD147 cells was significantly decreased compared with A549-pEGFP and A549-pSicoR cells, respectively (Fig. 3C).

Effects of CD147 on the expression of VEGF. The effect of CD147 on VEGF expression was analyzed using qPCR and western blot analysis. VEGF expression was decreased by $61.9 \%$ at the protein and $56.7 \%$ at the mRNA level in A549siCD147 cells $(\mathrm{P}=0.0082)$ and increased by $39.96 \%$ at the protein and $37.55 \%$ at the mRNA level in A549-CD147 cells $(\mathrm{P}<0.01)$ compared with A549 cells (Fig. 4A and B).

Effects of CD147 on the angiogenesis in endothelial cells. The number of vascular formations made by HUVEC in the A549-CD147 supernatant was significantly increased compared with A549-pEGFP $(\mathrm{P}<0.01)$. By contrast, the number of vascular formations made by HUVEC in the A549-siCD147 supernatant was significantly decreased compared with A549-pSicoR ( $\mathrm{P}=0.0079)$ (Fig. 4C).

\section{Discussion}

Tumor cells undergo a complex multi-step process with multi-factor involvement during proliferation and infiltration, including the degradation of basement membrane, stroma infiltration, migration out of blood vessels and invasion of target tissues (16). Although there are a variety of tumor types, there are always significant similar biological characteristics that tumors share that promote growth and development (17). High expression of CD147 in tumor cells increases the expression and activity of MMPs, which in turn degrade the main component of the basement membrane, damage compartmentalization barriers and contribute to tumor invasion and metastasis (4). In addition, MMPs induce the activity of VEGF, which is originally released from the extracellular matrix in a non-active form. VEGF, a specific activator of endothelial cells, increases the permeability of small blood vessels, which results in leakage of plasma proteins and the formation of extra vascular fibrin gel. This not only provides a suitable environment for the growth of endothelial cells, but also increases the pressure within the tumor organization, impedes the delivery of nutrients, oxygen and therapeutic agents to the tumor and stimulates the growth of novel blood vessels $(18,19)$. Therefore, regulating VEGF may directly affect the formation of tumor blood vessels.

The present study firstly investigated the expression of CD147 and VEGF in human lung carcinoma A549 cells, and the results revealed that the expression of the two proteins were increased. This is the same as results demonstrated previously using immunohistochemistry in malignant melanoma (20). Subsequent immunofluorescence expression detection performed in the present study also obtained similar results. This provided the basis for the present study to additionally 

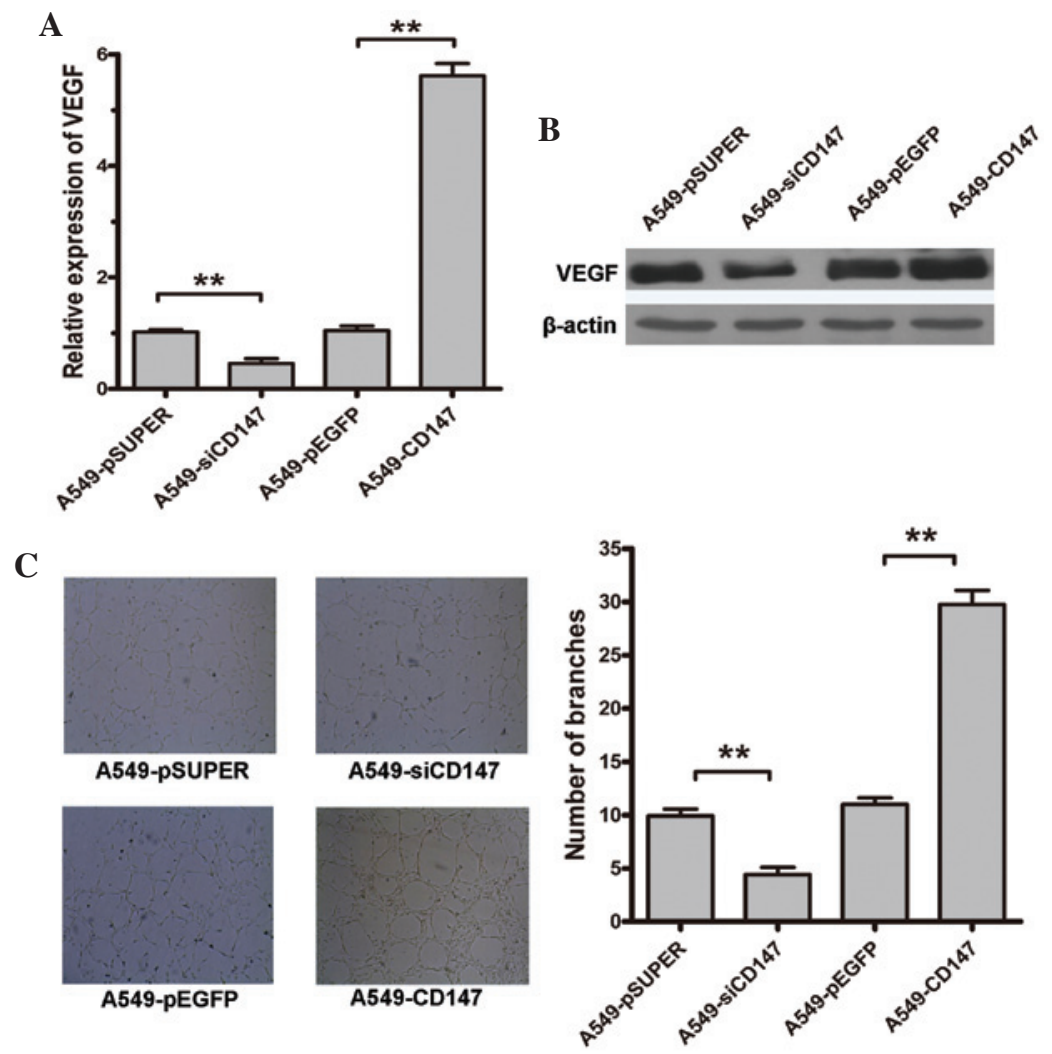

Figure 4. (A and B) Expression of VEGF in A549-CD147, A549-pEGFP, A549-siCD147 and A549-pSicoR cells as detected by (A) quantitative polymerase chain reaction and (B) western blot analysis. (C) Vascular formations of human umbilical vein endothelial cells in the supernatant of A549-CD147, A549-pEGFP, A549-siCD147 and A549-pSicoR. ${ }^{* *} \mathrm{P}<0.01$. CD, cluster of differentiation; VEGF, vascular endothelial growth factor.

investigate the function of CD147 and the association between CD147 and VEGF in tumor cells. In order to compare and analyze the function and role of CD147, the present study constructed four types of A549 cell lines: Stable overexpression of CD147, silencing of CD147 using siRNA and two empty vector cell lines as controls. Compared with a previous study, which used only a single cell line as a control group (21), the present results may be more reliable.

Tumor cells undergo significant cell proliferation; therefore, inhibiting the proliferation of cells is an important method for the treatment of cancer. In an in vitro study, Wang et al (22) demonstrated that silencing the expression of CD147 using RNA interference in gastric cancer SGC7901 cells decreased cell proliferation. Similarly, the present results reveled that the rate of cell proliferation in cells overexpressing CD147 was significantly increased compared with other cell types, including the siRNA-CD147 group of cells that had the slowest rate of proliferation. Consequently, it may be inferred that CD147 is a molecule that is important in lung carcinoma cell proliferation. These results suggest that the proliferative rate of lung carcinoma cells may be decreased by inhibiting the activity of CD147.

Invasion and metastasis are the most important biological characteristics of malignant tumors, present major challenges for clinical treatment and are the leading cause of mortality for patients with cancer (23). Therefore, inhibition of tumor invasion and metastasis is the most direct and effective way for the treatment of cancer. In the present study, cells that overexpressed CD147 had a great invasive ability in the Transwell-Matrigel assay, while siCD147 cells had a decreased invasion ability, which decreased the infiltration process. Results of subsequent scratch tests revealed that CD147 promoted the metastasis of lung carcinoma cells. This confirmed that CD147 is important in promoting invasion and metastasis of lung carcinoma cells, which are consistent with results demonstrated by Pan et al (24) in pancreatic cancer cells. The present results suggest that inhibiting the expression of CD147 may reduce the ability of lung carcinoma cells to invade and metastasize.

The formation of tumor angiogenesis is essential for the proliferation and growth of primary tumor cells, and is also required for invasion and metastasis of the tumor (25-27). The present study identified the expression of VEGF in cell lines at the mRNA and protein levels, and the results were similar to those demonstrated by Tang et al (28) in breast cancer cells: The level of VEGF expression increased and decreased in tandem with CD147 expression. Results of subsequent tube formation assays performed by the present study were also consistent with these results and demonstrated that CD147 regulates VEGF; compared with the control group, the number of tube formations in cells overexpressing CD147 was increased compared with siRNA-CD147 cells. These results reveal that CD147 efficiently upregulated the expression of VEGF and promoted angiogenesis of cells. Millimaggi et al (29) and Voigt et al (30) also demonstrated similar results in other tumor cell studies. VEGF is the most effective target factor for anti-angiogenic therapy, and the targeted drug bevacizumab is suggested in the National Comprehensive Cancer Network guidelines for first-line treatment of non-small cell lung cancer (www.ncnn.org/). However, 
although bevacizumab has been used as a clinical treatment, its clinical benefit is not satisfactory due to a lack of an effective tumor marker (31). Thus, identifying a reliable marker is extremely important (32). Due to CD147 overexpression in various tumors and its regulating affect on VEGF, studies have been conducted regarding the use of CD147 as a tumor marker. Preliminary results in studies in animal head and neck tumors revealed that a high expression of CD147 was associated with the best efficacy of bevacizumab (33).

In conclusion, the present preliminary findings demonstrated that CD147 may be important in promoting lung carcinoma cell proliferation, invasion and metastasis, and may increase the expression of VEGF, thus stimulating tumor angiogenesis. Consequently, these results have implications for CD147 as a potential target for tumor treatment. The present study was only conducted in one cell line; therefore, additional study is required to investigate other pathological cell lines to demonstrate a consistent validation. In depth future study by the present authors will continue to investigate the functional mechanism and regulation of CD147.

\section{References}

1. Johnson NB, Hayes LD, Brown K, Hoo EC and Ethier, KA; Centers for Disease Control: CDC National Health Report: Leading Causes of Morbidity and Mortality and Associated Behavioral Risk and Protective Factors - United States, 2005-2013. Morbidity and Mortality Weekly Report 63: 3-27, 2014.

2. Kadara H, Kabbout M and Wistuba II: Pulmonary adenocarcinoma: A renewed entity in 2011. Respirology 17: 50-65, 2012.

3. Biswas C, Zhang Y, Decastro R, Guo H, Nakamura T, Kataoka H and Nabeshima K: The human tumor cell-derived collagenase stimulatory factor (renamed EMMPRIN) is a member of the immunoglobulin supperfamily.Cancer Res 55, 434-439, 1995.

4. Kanekura T, Chen X and Kanzaki T: Basigi (CDI47) is expressed on melanoma cells and induces tumor cell invasion by stimulating production of matrix metalloproteinases by fibroblasts. Int J Cancer 99: 520-528, 2002.

5. Li R, Huang L, Guo H and Toole BP: Basigin (murine EMMPRIN) stimulates matrix metalloproteinase production by fibroblasts. J Cell Physiol 186: 371-379, 2001.

6. Sameshima T, Nabeshima K, Toole BP, Yokogami K, Okada Y, Goya T, Koono M and Wakisaka S: Glioma cell extracellular matrix metalloproteinase inducer (EMMPRIN) (CD147) stimulates production of membrane-type matrix metalloproteinases and activated gelatinase $\mathrm{A}$ in co-cultures with brain-derived fibroblasts. Cancer Lett 157: 177-184, 2000.

7. Muramatsu T and Miyauchi T: Basigin (CD147): A multifunctional transmembrane protein involved in reproduction, neural function, inflammation and tumor invasion. Histol Histopathol 18: 981-987, 2003.

8. Nabeshima K, Iwasaki H, Koga K, Hojo H, Suzumiya J and Kikuchi M: Emmprin (basigin/CD147): Matrix metalloproteinase modulator and multifunctional cell recognition molecule that plays a critical role in cancer progression. Pathol Int 56: 359-367, 2006.

9. Kanekura $\mathrm{T}$ and Chen X: CD147/basigin promotes progression of malignant melanoma and other cancers. J Dermatol Sci 57: 149-154, 2010.

10. Chen X, Lin J, Kanekura T, Su J, Lin W, Xie H, Wu Y, Li J, Chen M and Chang J: A small interfering CD147-targeting RNA inhibited the proliferation, invasiveness, and metastatic activity of malignant melanoma. Cancer Res 66: 11323-11330, 2006.

11. Hao JL, Cozzi PJ, Khatri A, Power CA and Li Y: CD147/EMMPRIN and CD44 are potential therapeutic targets for metastatic prostate cancer. Curr Cancer Drug Targets 10 287-306, 2010

12. Chen X, Kanekura T, Tsuyama S, Murata F and Kanzaki T: Ultrastructural localization of basigin in normal human epidermis Histochem Cell Biol 115: 465-470, 2001.
13. Chen X, Kanekura T and Kanzaki T: Expression of basigin in human fetal, infantile and adult skin and in basal cell carcinoma. J Cutan Pathol 28: 184-190, 2001.

14. Yuasa J, Toyama Y, Miyauchi T, Maekawa M, Yuasa S and Ito H: Specific localization of the basigin protein in human testes from normal adults, normal juveniles, and patients with azoospermia. Andrologia 33: 293-299, 2001.

15. Livak KJ and Schmittgen TD: Analysis of relative gene expression data using real-time quantitative PCR and the 2(-Delta Delta C(T)) method. Methods 25: 402-408, 2001.

16. Heike V, Vetter-Kauczok CS, Schrama D, Hofmann UB, Becker JC and Houben R: CD147 impacts angiogensis and metastasis formation. Cancer Invest 27: 329-333, 2009.

17. Shibuya M: Vascular endothelial growth factor-dependent and-independent regulation of angiogenesis. BMB Rep 41: 278-286, 2008

18. Ranieri G and Gasparini G: Angiogenesis and angiogenesis inhibitors: A new potential anticancer therapeutic strategy. Curr Drug Targets Immune Endocr Metabol Disord 1: 241-253, 2001.

19. Hicklin DJ and Ellis LM: Role of the vascular endothelial growth factor pathway in tumor growth and angiogenesis. J Clin Oncol 23: 1011-1027, 2005.

20. Kanekura T and Chen X: CD147 promotes progression of malignant melanoma and other cancers. J Dermatol Sci 57: 149-154, 2010.

21. Tang J1, Wu YM, Zhao P, Yang XM, Jiang JL and Chen ZN: Overexpression of HAB18G/CD147 promotes invasion and metastasis via $\alpha 3 \beta 1$ integrin mediated FAK-paxillin and FAK-PI3K-Ca2 pathways. Cell Mol Life Sci 65: 2933-2942, 2008.

22. Wang B, Xu YF, He BS, Pan YQ, Zhang LR, Zhu C, Qu LL and Wang SK: RNAi-mediated silencing of CD147 inhibits tumor cell proliferation, invasion and increase chemosensitivity to cisplatin in SGC7901 cells in vitro. J Exp Clin Cancer Res 29: 61-69, 2010.

23. Bareschino MA, Schettino C, Rossi A, Maione P, Sacco PC, Zeppa R and Gridelli C: Treatment of advanced non small cell lung cancer. J Thorac Dis 3: 122-133, 2011.

24. Pan Y, He B, Song G, Bao Q, Tang Z, Tian F and Wang S: CD147 silencing via RNA interference reduces tumor cell invasion, metastasis and increase chemosensitivity in pancreatic cancer cells. Oncol Rep 27: 2003-2009, 2012.

25. Yang X, Zhang P, Ma Q, Kong L, Li Y, Liu B and Lei D: EMMPRIN silenceing inhibits proliferation and perineural invasion of human salivary adenoid cystic carcinoma cells in vitro and in vivo. Cancer Biol Ther 13: 85-91, 2012.

26. Jain RK, Duda DG, Clark JW and Loeffler JS: Lessons from phase III clinical trails on anti-VEGF therapy for cancer. Nat Clin Pract Oncol 3: 24-40, 2006.

27. Jia L, Cao J, Wei W, Wang S, Zuo Y and Zhang J: CD147 depletion down-regulates matrix metalloproteinase-11, vascular endothelial growth factor-A expression and the lymphatic metastasis potential of murine hepatocarcinoma Hca-F cells. Int J Biochem Cell Biol 39: 2135-2142, 2007.

28. Tang Y, Nakada MT, Kesavan P, McCabe F, Millar H, Rafferty P, Bugelski P and Yan L: Extracellular matrix metalloproteinase inducer stimulates tumor angiogensis by elevating vascular endothelial cell growthfactor and matrix metalloproteinases. Cancer Res 65: 3193-3199, 2005.

29. Millimaggi D, Mari M, D'Ascenzo S, Carosa E, Jannini EA, Zucker S, Carta G, Pavan A and Dolo V: Tumor vesicle-associated CD147 modulates the angiogenic capability of endothelial cells. Neoplasia 9: 349-357, 2007.

30. Voigt H, Vetter-Kauczok CS, Schrama D, Hofmann UB, Becker JC and Houben R: CD147 impacts angiogensis and metastasis formation. Cancer Invest 27: 329-333, 2009.

31. Zhong J1, Ali AN, Voloschin AD, Liu Y, Curran WJ Jr, Crocker IR and Shu HK: Bevacizumab-induced hypertension is a predictive marker for improved outcomes in patients with recurrent glioblastoma treated with bevacizumab. Cancer 121: 1456-1462, 2015.

32. Crino L, Dansin E, Garrido P, Griesinger F, Laskin J, Pavlakis N, Stroiakovski D, Thatcher N, Tsai CM, Wu YL and Zhou C: Safety and efficacy of first-line bevacizumab-based therapy in advanced non-squamous non-small-cell lung cancer (SAiL, MO19390): A phase 4 study. Lancet Oncol 11: 733-740, 2010.

33. Newman JR, Helman EE, Safavy S, Zhang W and Rosenthal EL: EMMPRIN expression is required for response to bevacizumab therapy in HNSCC xenografts. Cancer Lett 274: 313-318, 2009. 